TRANSACTIONS OF THE

AMERICAN MATHEMATICAL SOCIETY

Volume 364, Number 8, August 2012, Pages 4155-4168

S 0002-9947(2012)05454-7

Article electronically published on March 21, 2012

\title{
SMASH PRODUCTS AND DIFFERENTIAL IDENTITIES
}

\author{
CHEN-LIAN CHUANG AND YUAN-TSUNG TSAI
}

To Pjek-Hwee Lee on his retirement

\begin{abstract}
Let $\mathbf{U}$ be the universal enveloping algebra of a Lie algebra and $R$ a $\mathbf{U}$-module algebra, where $\mathbf{U}$ is considered as a Hopf algebra canonically. We determine the centralizer of $R$ in $R \# \mathbf{U}$ with its associated graded algebra. We then apply this to the Ore extension $R[X ; \phi]$, where $\phi: X \rightarrow \operatorname{Der}(R)$. With the help of PBW-bases, the following is proved for a prime $\operatorname{ring} R$ : Let $Q$ be the symmetric Martindale quotient ring of $R$. For $f_{i}, g_{i} \in Q[X ; \phi], \sum_{i} f_{i} r g_{i}=0$ for all $r \in R$ iff $\sum_{i} f_{i} \otimes g_{i}=0$, where $\otimes$ is over the centralizer of $R$ in $Q[X ; \phi]$. Finally, we deduce from this Kharchenko's theorem on differential identities.
\end{abstract}

\section{INTRODUCTION}

By a derivation of an associative ring $R$, not necessarily with 1 , we mean a map $\delta: R \rightarrow R$ satisfying

$$
\delta(x+y)=\delta(x)+\delta(y), \quad \delta(x y)=\delta(x) y+x \delta(y) \quad \text { for } x, y \in R .
$$

Given $a \in R$, define the $\operatorname{map} \operatorname{ad}(a): R \rightarrow R$ by $r \in R \mapsto a r-r a$. We check easily that $\operatorname{ad}(a)$ is a derivation of $R$, called the inner derivation defined by $a \in$ $R$. Let $\operatorname{Der}(R)$ denote the set of derivations of $R$ and $\operatorname{Der}_{0}(R)$ the set of inner derivations of $R$. Clearly, $\operatorname{Der}(R)$ forms a Lie ring with respect to $[\delta, d] \stackrel{\text { def. }}{=} \delta d-d \delta$ for $\delta, d \in \operatorname{Der}(R)$. Also clearly, $\operatorname{Der}_{0}(R)$ forms a Lie ideal of $\operatorname{Der}(R)$ in the sense that $\left[\operatorname{Der}(R), \operatorname{Der}_{0}(R)\right] \subseteq \operatorname{Der}_{0}(R)$.

Our primary aim is to investigate differential identities of a prime ring $R$ in terms of Ore extensions (to be defined in $\S 3$ ), as initiated in Amitsur 1 for a single derivation and extended in [5] to a set of derivations. For this purpose, we have to compute the centralizer of $R$ in the Ore extension. This was done for Ore extensions with one indeterminate in 1 for simple rings and was extended to prime rings in [9. The crucial computation of [1] was interpreted in terms of Hasse-Schmidt higher derivations. For Ore extensions with many indeterminates, the computation of the centralizer of $R$ was left open in [5]. Higher derivations don't help much here because of the lack of the division algorithm. Surprisingly, it turns out that this can be done much easier in the more general context of smash products (to be explained in $\S 2$ ) with the associated graded algebras. We apply this to Ore extensions in $\S 3$ and then deduce in $\S 4$ an interpretation of Kharchenko's theory of differential identities in the context of Ore extensions. It seems very interesting whether results of $\S 4$ can be extended to the context of smash products

Received by the editors May 4, 2010 and, in revised form, August 30, 2010.

2010 Mathematics Subject Classification. Primary 16S40, 16S32, 16W25, 16S36, 16 S30.

Key words and phrases. Derivations, universal enveloping algebras, centralizers, smash products, Ore extensions, differential identities. 
considered in $\S 2$. Furthermore, can all these be generalized to $q$-skew derivations or skew derivations $([4,8])$ ?

\section{SMASH PRODUCTS}

Throughout here, $k$ is a field. An associative (or Lie, Hopf) algebra over $k$ will be called an associative (or Lie, Hopf resp.) $k$-algebra. By a Lie ring or a Lie algebra $\mathfrak{g}$, we always mean a restricted $p$ Lie ring or algebra if char $k=p \geq 2$.

Let $\mathfrak{g}$ be a Lie $k$-algebra. It is well known that the universal enveloping algebra of $\mathfrak{g}$, denoted by $\mathbf{U}$, forms a pointed irreducible cocommutative Hopf algebra with respect to the comultiplication $\Delta(a)=a \otimes 1+1 \otimes a$, the counit $\varepsilon(a)=0$ and the antipode $S(a) \stackrel{\text { def. }}{=}-a$ for $a \in \mathfrak{g}$. Assume that $R$ is an associative $k$-algebra. Let $\operatorname{Der}_{k}(R)$ be the set of $k$-linear derivations of $R$ and $\operatorname{End}_{k}(R)$ the set of $k$-linear maps of $R$. So $\operatorname{Der}_{k}(R)$ forms a Lie $k$-algebra and $\operatorname{End}_{k}(R)$ forms an associative $k$-algebra. Let $\phi: \mathfrak{g} \rightarrow \operatorname{Der}_{k}(R)$ be a Lie $k$-algebra homomorphism. By the universal mapping property, $\phi$ extends uniquely to a $k$-algebra homomorphism $\mathbf{U} \rightarrow \operatorname{End}_{k}(R)$, also denoted by $\phi$. With respect to $\phi$ thus extended, the $k$-algebra $R$ is a $\mathbf{U}$-module algebra and we can form the smash product $R \# \mathbf{U}$. This is the $k$-space $R \otimes_{k} \mathbf{U}$, where we write $a \otimes h$ as $a \# h$ for $a \in R$ and $h \in \mathbf{U}$, endowed with multiplication defined by

$$
(a \# h)(b \# g)=a b \# h g+a \phi(h)(b) \# g \quad \text { for } a, b \in R \text { and } h, g \in \mathbf{U} .
$$

We refer the reader to 10 and 13 for the details. Our aim here is to describe the centralizer of $R$ in $R \otimes_{k} \mathbf{U}$. We recall the following:

Definition 1. Let $R$ be a $k$-algebra with 1 and ${ }_{R} M$ be a left $R$-module. We call a finite sum $\sum_{i} a_{i} m_{i}$, where $a_{i} \in R$ and $m_{i} \in M$, a left $R$-linear combination of $m_{i}$. By a left $R$-basis of ${ }_{R} M$, we mean a subset $B$ of $M$ such that any $m \in M$ can be uniquely written as a left $R$-linear combination of elements in $B$. A right $R$-basis of a right $R$-module is defined analogously. By an $R$-basis of an $(R, R)$-bimodule ${ }_{R} M_{R}$, we mean a subset $B$ of $M$ which is both a left $R$-basis of ${ }_{R} M$ and a right $R$-basis of $M_{R}$.

The well-known Poincaré-Birkhoff-Witt Theorem asserts that regular words in a linearly ordered $k$-basis of $\mathfrak{g}$ form a $k$-basis of its universal enveloping $k$-algebra $\mathbf{U}$ and hence form an $R$-basis of $R \# \mathbf{U}$. But our main concern is the centralizer of $R$ in $R \# \mathbf{U}$. Let $C$ be the center of $R$. Clearly, $C$ centralizes $R$. So the dependence of elements of $\mathfrak{g}$ over $C$, not merely over the subfield $k$ of $C$, has to be considered. We recall the notion of regular words in this general context as follows.

Definition 2. Given a set $B$, elements of the free monoid generated by $B$ are called $B$-words. The identity of the free monoid generated by $B$, denoted by 1 , is called the empty $B$-word. Given a $B$-word, write

$$
W=b_{1} b_{2} \cdots b_{n},
$$

where $b_{i} \in B$ and where we postulate $W=1$ for convenience if $n=0$. We call $n$ the $B$-length of $W$ and write $\operatorname{lh}_{B}(W)=n$. Assume further that $B$ is linearly ordered by $<$. By a regular $B$-word, we mean a $B$-word in the form

$$
b_{1}^{n_{1}} b_{2}^{n_{2}} \cdots b_{s}^{n_{s}}
$$


where the $b_{i} \in B$ satisfy $b_{1}<b_{2}<\cdots<b_{s}$ and, in the case of char $k=p \geq 2$, where $0<n_{i}<p$ for each $i$. We order regular $B$-words first by length and then lexicographically for $B$-words of the same length.

We shall apply the above notions to algebras. To be precise, we state the following.

Definition 3. Let $A$ be an associative $k$-algebra with 1 and $B$ a subset of $A$. By a $B$-product, we mean an expression in $A$ of the form

$$
b_{1} \cdot b_{2} \cdot b_{3} \cdots b_{n}
$$

where $b_{i} \in B$ and where - denotes the multiplication of $A$. For simplicity of terminology and as an abuse of language, whenever there is no confusion, the $B$-product above will be identified with the $B$-word $W \stackrel{\text { def. }}{=} b_{1} b_{2} \cdots b_{n}$ in the free monoid generated by $B$ (as a set of symbols). So the above $B$-product is regular if so is $W$ and so on.

We stress here that the above notions apply not to elements of $A$ but to expressions of elements of $A$ as products of elements of $B$. An element of $A$ may be expressed as many $B$-products with different associated $B$-words of different $B$-lengths and we have to know which expression is meant. Regular words occur naturally in the following.

Lemma 1. Let $R$ be a $k$-algebra with 1 and $R[Y]$ the $k$-algebra generated by $R$ and a set $Y$ of commuting indeterminates subjected to $y r=r y$ and $y y^{\prime}=y^{\prime} y$ for $r \in R$ and $y, y^{\prime} \in Y$. Set $R_{p}[Y] \stackrel{\text { def. }}{=} R[Y] / I$, where $I$ is the ideal of $R[Y]$ defined by

$$
I \stackrel{\text { def. }}{=} \begin{cases}0 & \text { if char } k=0, \\ \text { the ideal generated by } y^{p} \text { for } y \in Y & \text { if char } k=p>0 .\end{cases}
$$

For any linear order $<$ of $Y$, regular $Y$-words form an $R$-basis of the polynomial ring $R_{p}[Y]$. More specifically, for each $n \geq 0$, regular $Y$-words of $Y$-length $n$ form an $R$-basis of the $R$-module of polynomials of degree $n$ in $R_{p}[Y]$.

Proof. This is obvious by the commutativity $y r=r y$ and $y y^{\prime}=y^{\prime} y$ for $r \in R$ and $y, y^{\prime} \in Y$.

Theorem 2. Assume that $R$ is a ring with the center $C$ being a field. Let $k$ be $a$ subfield of $C$ and $\mathfrak{g}$ a Lie $k$-algebra with the universal enveloping algebra $\mathbf{U}$. Assume that $R$ is a $\mathbf{U}$-module $k$-algebra. Let $B$ be a $C$-basis of $C \mathfrak{g}$ as a left $C$-subspace of the smash product $R \# \mathbf{U}$. For each $h \in B$, set $h^{\prime} \stackrel{\text { def. }}{=} h+a_{h}$, where $a_{h} \in R$ is arbitrarily chosen. Define $B^{\prime} \stackrel{\text { def. }}{=}\left\{h^{\prime}: h \in B\right\}$ and let $<$ be an arbitrary linear order of $B^{\prime}$. Then the set of regular $B^{\prime}$-words forms an $R$-basis of $R \# \mathbf{U}$.

Proof. For $n \geq 0$, let $V_{n}$ be the set of $f \in R \# \mathbf{U}$ which can be written in a finite sum $f=\sum_{i} a_{i} W_{i}$, where $0 \neq a_{i} \in R$ and where $W_{i}$ is a $\mathfrak{g}$-word of length $\leq n$ for each $i$. Clearly, $V_{n} V_{m} \subseteq V_{n+m}$ and

$$
V_{0} \subseteq V_{1} \subseteq V_{2} \subseteq \cdots
$$

So the associative $k$-algebra $R \# \mathbf{U}$ is filtered. Set $V_{-1} \stackrel{\text { def. }}{=} 0$ for convenience. Define $\bar{V}_{n} \stackrel{\text { def. }}{=} V_{n} / V_{n-1}$ for $n \geq 0$. For $\bar{a} \in \bar{V}_{n}$ and $\bar{b} \in \bar{V}_{m}$, where $a \in V_{n}$ and $b \in V_{m}$, 
define $\bar{a} \bar{b} \stackrel{\text { def. }}{=} \overline{a b} \in \bar{V}_{n+m}$. This is well-defined because of $V_{s} V_{t} \subseteq V_{s+t}$. With this, we form the graded $k$-algebra

$$
\operatorname{gr}(R \# \mathbf{U}) \stackrel{\text { def. }}{=} \bigoplus_{i \geq 0} \bar{V}_{i}
$$

We say that $f \in R \# \mathbf{U}$ has $\mathfrak{g}$-degree $n$ and write $\operatorname{deg}_{\mathfrak{g}}(f)=n$ if $f \in V_{n}-V_{n-1}$. Given $f \in R \# \mathbf{U}$ with $\operatorname{deg}_{\mathfrak{g}}(f)=n$, define

$$
\bar{f} \stackrel{\text { def. }}{=} f+V_{n-1} \in \bar{V}_{n} \subseteq \operatorname{gr}(R \# \mathbf{U}) .
$$

Given finitely many $f, f_{i} \in R \# \mathbf{U}$, if $f=f_{1}+f_{2}+\cdots$ and if $\operatorname{deg}_{\mathfrak{g}}(f)=\operatorname{deg}_{\mathfrak{g}}\left(f_{1}\right)=$ $\operatorname{deg}_{\mathfrak{g}}\left(f_{2}\right)=\cdots$, then clearly

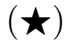

$$
\bar{f}=\bar{f}_{1}+\bar{f}_{2}+\cdots .
$$

For $S \subseteq R \# \mathbf{U}$, set $\bar{S} \stackrel{\text { def. }}{=}\{\bar{f} \in \operatorname{gr}(R \# \mathbf{U}): f \in S\}$. Let $h \in \mathfrak{g} \mapsto \delta_{h} \in \operatorname{Der}_{k}(R)$ be the Lie homomorphism $\mathfrak{g} \rightarrow \operatorname{Der}_{k}(R)$. For any $r \in R$ and $h \in \mathfrak{g}, h r-r h=\delta_{h}(r) \in R$ and hence $\bar{h} \bar{r}=\bar{r} \bar{h}$ in $\operatorname{gr}(R \# \mathbf{U})$. Since $\delta(C) \subseteq C$ for $\delta \in \operatorname{Der}(R), C \mathfrak{g}$ forms a Lie ring. So for $f_{1}, f_{2} \in C \mathfrak{g}, f_{1} f_{2}-f_{2} f_{1} \in C \mathfrak{g} \subseteq V_{1}$ and hence $\bar{f}_{1} \bar{f}_{2}=\bar{f}_{2} \bar{f}_{1}$ in $\operatorname{gr}(R \# \mathbf{U})$. Thus $\overline{C \mathfrak{g}}$ is a commuting set in $\operatorname{gr}(R \# \mathbf{U})$. Clearly, $\operatorname{gr}(R \# \mathbf{U})$ is the $k$-algebra generated by $R$ and the commuting set $\overline{C \mathfrak{g}}$.

Set $p \stackrel{\text { def. }}{=}$ char $R \geq 0$. Given a $C$-basis $Y$ of $\overline{C \mathfrak{g}}$, let $R_{p}[Y]$ be as defined in Lemma 1. We claim that there is a $k$-algebra homomorphism $\theta: R_{p}[Y] \rightarrow \operatorname{gr}(R \# \mathbf{U})$ such that $\theta(r)=r$ for $r \in R$ and such that $\theta(y)=y \in \bar{V}_{1}$ for $y \in Y$. This is clear if char $R=p=0$, for $R_{p}[Y] \stackrel{\text { def. }}{=} R[Y]$ is the freest $k$-algebra generated by $R$ and the commuting set $Y$. Assume $\operatorname{char} R=p \geq 2$. Since $\mathfrak{g}$ is a restricted $p$-Lie algebra by our convention, there is a unary $p$-operation $h \mapsto h^{[p]}$ for $h \in \mathfrak{g}$ such that in the universal enveloping algebra $\mathbf{U}$ we have

$$
\underbrace{h \cdot h \cdot h \cdots h}_{p \text { times }}=h^{p}=h^{[p]} \text {. }
$$

Since $h^{[p]} \in V_{1}$, we have $\bar{h}^{p}=0$ for $h \in \mathfrak{g}$ in the associated graded algebra $\operatorname{gr}(R \# \mathbf{U})$. Given $0 \neq f \in C \mathfrak{g}$, write $f=\alpha_{1} h_{1}+\alpha_{2} h_{2}+\cdots$, where $\alpha_{i} \in C$ and $h_{i} \in \mathfrak{g}$. By $(\star)$, $\bar{f}=\bar{\alpha}_{1} \bar{h}_{1}+\bar{\alpha}_{2} \bar{h}_{2}+\cdots$ and hence

$$
\bar{f}^{p}=\left(\bar{\alpha}_{1} \bar{h}_{1}+\bar{\alpha}_{2} \bar{h}_{2}+\cdots\right)^{p}=\bar{\alpha}_{1}^{p} \bar{h}_{1}^{p}+\bar{\alpha}_{2}^{p} \bar{h}_{2}^{p}+\cdots=0 .
$$

Since $Y \subseteq \overline{C \mathfrak{g}}$, we see that $y^{p}=0$ for $y \in Y$ in $\operatorname{gr}(R \# \mathbf{U})$. By the definition in Lemma 1, $R_{p}[Y]$ is the freest $k$-algebra generated by $R$ and the commuting set $Y$ subjected to the condition $y^{p}=0$ for $y \in Y$. The claim is thus proved.

We show that $\theta$ above is the $k$-algebra isomorphism of $R_{p}[Y]$ and $\operatorname{gr}(R \# \mathbf{U})$. The map $\theta$ is surjective, since $\operatorname{gr}(R \# \mathbf{U})$ is the $k$-algebra generated by $R$ and the commuting set $Y$. To show the injectivity of $\theta$, pick arbitrarily a $k$-basis $B$ of $\mathfrak{g}$ with a linear order $<$. By the Poincaré-Birkhoff-Witt Theorem, regular $B$-words form a $k$-basis of $\mathbf{U}$ and hence form an $R$-basis of $R \# \mathbf{U}$, since $R \# \mathbf{U}$, as a left $C$-space, is the same as the left $C$-space $R \otimes_{k} \mathbf{U}$; also $B$ forms a $C$-basis of $C \mathfrak{g}$. So regular $B$-words of $B$-lengths $\leq n$ form an $R$-basis of $V_{n}$ for each $n \geq 0$. Particularly, $\bar{B}$ forms a $C$-basis of $\overline{C \mathfrak{g}}$. The injectivity of $R_{p}[\bar{B}] \rightarrow \operatorname{gr}(R \# \mathbf{U})$ follows. Given an arbitrary $C$-basis $Y$ of $\overline{C \mathfrak{g}}$, there exist $\alpha_{b}^{y}, \beta_{y}^{b} \in C$ for $b \in B$ and $y \in Y$ such that

$$
y=\sum_{b \in B} \alpha_{b}^{y} \bar{b} \quad \text { and } \quad \bar{b}=\sum_{y \in Y} \beta_{y}^{b} y .
$$


Since both $Y$ and $\bar{B}$ are $C$-bases of $\overline{C \mathfrak{g}}$, the two expressions above are inverse to each other. The injectivity of $R_{p}[Y] \rightarrow \operatorname{gr}(R \# \mathbf{U})$ follows from that of $R_{p}[\bar{B}] \rightarrow$ $\operatorname{gr}(R \# \mathbf{U})$.

Let $B$ be a given left $C$-basis of $C \mathfrak{g}$. Clearly, $\bar{B}$ forms a $C$-basis of $\overline{C \mathfrak{g}}$ and hence $\operatorname{gr}(R \# \mathbf{U})=R_{p}[\bar{B}]$. Let $B^{\prime} \stackrel{\text { def. }}{=}\left\{h^{\prime}: h \in B\right\}$, where, for each $h \in B, h^{\prime} \stackrel{\text { def. }}{=} h+a_{h}$ for some $a_{h} \in R$. Given a linear order $<$ of $B^{\prime}$, we denote the corresponding linear order of $B$ also by $<$. By Lemma 1, regular $\bar{B}$-words of length $n$ form an $R$-basis for $\bar{V}_{n}$. For any $h_{1}, \ldots, h_{n} \in B$, where $n \geq 1$, we have $h_{1} \cdots h_{n} \equiv h_{1}^{\prime} \cdots h_{n}^{\prime}$ modulo $V_{n-1}$. So regular $\overline{B^{\prime}}$-words of length $n$ also form an $R$-basis for $\bar{V}_{n}$. With this, we see inductively that regular $B^{\prime}$-words of length $\leq n$ form a left $R$-basis of nonzero $V_{n}$. From this, our assertion follows.

Theorem 2 provides very good bases, which deserve a special definition below because of frequent uses in the sequel.

Definition 4. Let $R, C, k$ and $\mathfrak{g}$ be as in Theorem 2, Let $\phi: h \in C \mathfrak{g} \mapsto \delta_{h} \in$ $\operatorname{Der}_{k}(R)$ be the left $C$-linear map extending the Lie homomorphism $\mathfrak{g} \rightarrow \operatorname{Der}_{k}(R)$. $\left(\right.$ So $\phi(C \mathfrak{g})=C \phi(\mathfrak{g})$.) Set $\mathfrak{g}_{0} \stackrel{\text { def. }}{=}\left\{h \in C \mathfrak{g}: \delta_{h} \in \operatorname{Der}_{0}(R)\right\}$. Let $B$ be a left $C$-basis of $C \mathfrak{g}$ such that $B_{0} \stackrel{\text { def. }}{=} B \cap \mathfrak{g}_{0}$ forms a left $C$-basis of $\mathfrak{g}_{0}$. For each $h \in B_{0}$, pick $a_{h} \in R$ arbitrarily such that $\delta_{h}=\operatorname{ad}\left(a_{h}\right)$. Define $B_{0}^{\prime} \stackrel{\text { def. }}{=}\left\{h-a_{h}: h \in B_{0}\right\}$ and $B^{\prime} \stackrel{\text { def. }}{=} B_{0}^{\prime} \cup\left(B-B_{0}\right)$. Let $<$ be a linear order of $B^{\prime}$ such that

$$
h<g \quad \text { for } h \in B_{0}^{\prime} \text { and } g \in B-B_{0} .
$$

We call $B^{\prime}$ so ordered a regular Lie basis of the smash product $R \# \mathbf{U}$.

With regular Lie bases, we are able to compute the centralizer of $R$ in $R \# \mathbf{U}$. For latter applications, we have to characterize subsets $T$ of $R$ such that the centralizer of $T$ in $R \# \mathbf{U}$ is equal to the centralizer of $R$ in $R \# \mathbf{U}$. This seems interesting in itself.

Theorem 3. Let $R, C, k$ and $\mathfrak{g}$ be as in Theorem 2, Set $S \stackrel{\text { def. }}{=} R \# \mathbf{U}$. For $T \subseteq R$, set $C_{S}(T) \stackrel{\text { def. }}{=}$ the centralizer of $T$ in $S$.

(1) For $T \subseteq R, C_{S}(T)=C_{S}(R)$ iff for any $\delta \in C \phi(\mathfrak{g})+\operatorname{Der}_{0}(R), \delta(T)=0$ implies $\delta(R)=0$.

(2) Let $B^{\prime}$ be a regular Lie basis of $S$ and retain the notation of Definition 4 , The set of regular $B_{0}^{\prime}$-words forms a $C$-basis of the free $C$-module $C_{S}(R)$. For any $C$-basis $V$ of $R$, the set

$$
\mathbb{B} \stackrel{\text { def. }}{=}\left\{v W: v \in V \text { and } W \text { is a regular word in } B-B_{0}\right\}
$$

forms a $C_{S}(R)$-basis of the free $C_{S}(R)$-module $R \# \mathbf{U}$.

(3) Any element of $S \otimes_{C_{S}(R)} S$ can be uniquely expressed in the form $\sum_{i} f_{i} \otimes g_{i}$, where $f_{i} \in R \# \mathbf{U}$ and where the $g_{i} \in \mathbb{B}$ are distinct.

Proof. Given $h \in C \mathfrak{g}$ and $a \in R, \delta_{h}+\operatorname{ad}(a)$ vanishes on a subset $T$ of $R$ iff $h+a \in C_{S}(T)$. So if $C_{S}(T)=C_{S}(R)$, then $\delta(T)=0$ implies $\delta(R)=0$ for $\delta \in C \phi(\mathfrak{g})+\operatorname{Der}_{0}(R)$. On the other hand, suppose that $\delta(T)=0$ implies $\delta(R)=0$ for any $\delta \in C \phi(\mathfrak{g})+\operatorname{Der}_{0}(R)$. Clearly, $C$ and $B_{0}^{\prime}$ are contained in $C_{S}(R)$ and hence in $C_{S}(T)$. By Theorem 2, regular $B^{\prime}$-words form a left $R$-basis of $R \# \mathbf{U}$. Any 
regular $B^{\prime}$-word is of the form $\xi W$, where $\xi$ is a regular $B_{0}^{\prime}$-word and $W$ is a regular $\left(B-B_{0}\right)$-word. So any $f \in R \# \mathbf{U}$ can be uniquely expressed in the form

$$
f=\sum_{i} \xi_{i} f_{i}
$$

where $\xi_{i}$ ranges over all distinct regular $B_{0}^{\prime}$-words and where $f_{i} \in R \# \mathbf{U}$ are left $R$-linear combinations of regular $\left(B-B_{0}\right)$-words. Suppose that $f \in C_{S}(T)$. Since all $\xi_{i}$ centralize $R$, we have for any $r \in R$,

$$
0=f r-r f=\sum_{i} \xi_{i}\left(f_{i} r-r f_{i}\right) .
$$

Since subwords of regular $\left(B-B_{0}\right)$-words are also regular $\left(B-B_{0}\right)$-words, each $f_{i} r-r f_{i}$ is also a left $R$-linear combination of regular $\left(B-B_{0}\right)$-words. So each $\xi_{i}\left(f_{i} r-r f_{i}\right)$ is a left $R$-linear combination of regular $B^{\prime}$-words starting with $\xi_{i}$. Since the $\xi_{i}$ are distinct for distinct $i$, so are the $B^{\prime}$-words involved in $\xi_{i}\left(f_{i} r-r f_{i}\right)$. It follows that $f_{i} r-r f_{i}=0$ for all $i$ and all $r \in T$. That is, $f_{i} \in C_{S}(T)$ for all $i$. All $f_{i}$ 's are left $R$-linear combinations of regular $\left(B-B_{0}\right)$-words. It thus suffices to show that for any left $R$-linear combination $g$ of regular $\left(B-B_{0}\right)$-words, if $g \in C_{S}(T)$, then $g \in C$. So consider such a $g$ and write it as a left $R$-linear combination of distinct regular $\left(B-B_{0}\right)$-words $W_{i}$ :

$$
g=a_{1} W_{1}+a_{2} W_{2}+\cdots, \quad \text { where } a_{i} \in R .
$$

If $W_{i}$ has the maximal length among all $W_{1}, W_{2}, \ldots$, then for any $r \in T$,

$$
0=g r-r g=\left(a_{i} r-r a_{i}\right) W_{i}+\cdots,
$$

where the dots denote a left $R$-linear combination of regular $\left(B-B_{0}\right)$-words distinct from $W_{i}$. So $a_{i} r-r a_{i}=0$ for any $r \in T$. That is, the inner derivation $\operatorname{ad}\left(a_{i}\right)$ vanishes on $T$ and hence on $R$ by our assumption of $T$. That is, $a_{i} \in C$. Let us assume that $W_{1}$ is the <-maximum among all $W_{i}$. If $W_{1} \neq \emptyset$, then write

$$
W_{1}=b_{1}^{n_{1}} b_{2}^{n_{2}} \cdots b_{s}^{n_{s}},
$$

where $b_{i} \in B-B_{0}$ satisfy $b_{1}<b_{2}<\cdots<b_{s}$ and where $0<n_{i}<p$ for each $i$ in the case of char $k=p \geq 2$. Suppose that

$$
W_{j}=b_{1}^{n_{1}-1} b_{2}^{n_{2}} \cdots b_{s}^{n_{s}} \quad \text { for some } j .
$$

Also, assume that $W_{2}, \ldots, W_{m}$ enumerate all those $W_{i}$ of maximal length such that $W_{i}=d_{i} W_{j}=d_{i} b_{1}^{n_{1}-1} b_{2}^{n_{2}} \cdots b_{s}^{n_{s}}$ for some $d_{i} \in B-B_{0}-\left\{b_{1}\right\}$. Recall that $\phi: h \in C \mathfrak{g} \mapsto \delta_{h} \in \operatorname{Der}_{k}(R)$ denotes the left $C$-linear map extending the Lie $k$-algebra homomorphism $\mathfrak{g} \rightarrow \operatorname{Der}_{k}(R)$. The left coefficient of $b_{1}^{n_{1}-1} b_{2}^{n_{2}} \cdots b_{s}^{n_{s}}$ in $g r-r g$ is then given by

$$
n_{1} a_{1} \delta_{b_{1}}(r)+\sum_{i=2}^{m} a_{i} \delta_{d_{i}}(r)+a_{j} r-r a_{j} .
$$

We have seen that $a_{i} \in C$ for $i=0,1, \ldots, m$. So the above expression defines a derivation in $C \phi(\mathfrak{g})+\operatorname{Der}_{0}(R)$. Since $g$ centralizes $T$, the above expression vanishes for $r \in T$ and hence for $r \in R$ by our assumption of $T$. So we have

$$
n_{1} a_{1} \delta_{b_{1}}+\sum_{i=2}^{m} a_{i} \delta_{d_{i}}+\operatorname{ad}\left(a_{j}\right)=0 .
$$


So $n_{1} a_{1} b_{1}+\sum_{i=2}^{m} a_{i} d_{i}$ falls in $C \mathfrak{g}_{0}$ and hence can be expressed as a $C$-linear combination of $B_{0}^{\prime}$. But $b_{1}, d_{2}, \ldots, d_{m} \in B-B_{0}$, implying $n_{1} a_{1}=0$. Also, $n_{1}$ is invertible in $R$. So $a_{1}=0$, a contradiction. (1) is thus proved. By $(*)$, any regular $B^{\prime}$-word can be uniquely written as a product $\xi W$, where $\xi$ is a regular $B_{0}^{\prime}$-word and $W$ is a regular $\left(B-B_{0}\right)$-word. By Theorem 2 , these words $\xi W$ form a left $R$-basis of $R \# \mathbf{U}$. Since $V$ is a $C$-basis of $R$, the set $v \xi W$, where $v \in V, \xi$ is a regular $B_{0}^{\prime}$-word and $W$ is a regular $\left(B-B_{0}\right)$-word, forms a $C$-basis of $R \# \mathbf{U}$. But $v \xi W=\xi v W$, since $\xi$ centralizes $R$. So (2) follows. As right $C_{S}(R)$-modules, $S=\bigoplus_{i} C_{S}(R) g_{i}$, where $g_{i}$ enumerate $\mathbb{B}$. So

$$
S_{C_{S}(R)}^{\otimes} S=S \underset{C_{S}(R)}{\otimes}\left(\bigoplus_{i} C_{S}(R) g_{i}\right)=\bigoplus_{i} S_{C_{S}(R)}^{\otimes} g_{i}
$$

So (3) follows.

\section{Ore EXtensions}

Given a set $X$ of noncommuting indeterminates, finite or infinite, and a map $\phi: X \rightarrow \operatorname{Der}(R)$, write $\delta_{x} \stackrel{\text { def. }}{=} \phi(x)$ for brevity. Let $R[X ; \phi]$ denote the ring of polynomials in indeterminates $x \in X$ and with coefficients in $R$ subjected to the following commutation rule for $a \in R$ and $x \in X$ :

$$
x a=a x+\delta_{x}(a), \text { where } \delta_{x}=\phi(x) \in \operatorname{Der}(R) .
$$

We call $R[X ; \phi]$ the Ore extension of $R$ by $\phi$. (See [3, 15, 14].) We stress here that the indeterminates $x \in X$ do not commute with each other and that the map $\phi$ may not be injective. So distinct $x \in X$ can be associated with the same derivation.

In traditional notation, we enumerate $X$ as a sequence $x_{i}, i=0,1, \ldots$, and let $D$ be the corresponding sequence $\delta_{i} \stackrel{\text { def. }}{=} \phi\left(x_{i}\right)=\delta_{x_{i}} \in \operatorname{Der}(R), i=0,1, \ldots$ In this way, the map $\phi$ is explicitly encoded in the two corresponding sequences $X$ and $D$. We can thus denote $R[X ; \phi]$ by $R[X ; D]$. Ore extensions are also called skew polynomial rings, which has become one of the most basic and useful constructions in ring theory. This topic has been extensively studied in various directions for a few decades.

Here are some interesting special instances of $R[X ; D]$ : If $X$ is a singleton, say $X=\{x\}$, then $R[X ; \phi]$ is commonly written as $R[x ; \delta]$, where $\delta \stackrel{\text { def. }}{=} \phi(x) \in \operatorname{Der}(R)$. This is the most extensively investigated Ore extension. If $\delta$ happens to be the zero derivation, then $R[x ; \delta]$, usually written as $R[x]$ and called the polynomial ring in $x$ over $R$, is merely the ring $R$ adjoined by the indeterminate $x$ which commutes with $R$. More generally, if $\phi(x)=0$ for $x \in X$, then the Ore extension $R[X ; \phi]$, usually denoted by $R\langle X\rangle$ and called the free algebra generated by $X$ over $R$, is simply the ring $R$ adjoined by the indeterminates $x \in X$ which all commute with $R$ but which do not commute with each other.

Let $R$ be an associative $k$-algebra and $\phi: X \rightarrow \operatorname{Der}_{k}(R)$. Let $\mathfrak{g}_{X}$ be the Lie $k$-algebra generated by $X$ in $R[X ; \phi]$. (So $\mathfrak{g}_{X}$ is a restricted $p$-Lie $k$-algebra if char $R=p \geq 2$ by our convention.) Clearly, $\mathfrak{g}_{X}$ is the free Lie $k$-algebra generated by $X$. By [12] or [11, the universal enveloping algebra of $\mathfrak{g}_{X}$ is $k\langle X\rangle$, the free associative $k$-algebra generated by $X$. This is also contained in $R[X ; \phi]$. The map $\phi: X \rightarrow \operatorname{Der}_{k}(R)$ extends to a unique Lie $k$-algebra homomorphism $\mathfrak{g}_{X} \rightarrow \operatorname{Der}_{k}(R)$ by the freedom of $\mathfrak{g}_{X}$ on the generator set $X$ and then to a $k$-algebra homomorphism $k\langle X\rangle \rightarrow \operatorname{End}_{k}(R)$ by the freedom of $k\langle X\rangle$. With the map thus extended, which 
we also denoted by $\phi, R$ is a $k\langle X\rangle$-module algebra. It was pointed out to us by the referee of [14 that the Ore extension $R[X ; \phi]$ can be interpreted as a smash product as the following.

Lemma 4. In the context above, the Ore extension $R[X ; \phi]$ is canonically isomorphic with the smash product $R \# k\langle X\rangle$ via the map $a \mapsto a \# 1$ for $a \in R$ and $x \mapsto 1 \# x$ for $x \in X$.

Proof. For $a \in R$ and $x \in X$, write $\delta_{x} \stackrel{\text { def. }}{=} \phi(x)$ and we have

$$
(1 \# x)(a \# 1)=a \# x+\phi(x)(a) \# 1=a \# x+\delta_{x}(a) \# 1 .
$$

So $a \mapsto a \# 1$ for $a \in R$ and $x \mapsto 1 \# x$ for $x \in X$ induces a surjective $k$-algebra homomorphism $R[X ; \phi] \rightarrow R \# k\langle X\rangle$. Suppose that $f \in R[X ; \phi]$ is in the kernel of the above $k$-algebra homomorphism. Write $f=\sum_{i} a_{i} w_{i}$, where $a_{i} \in R$ and where $w_{i}$ are distinct words in $X$. Then $0=\sum_{i} a_{i} \# w_{i}=\sum_{i} a_{i} \otimes_{k} w_{i}$. The distinct words $w_{i}$, as elements of $k\langle X\rangle$, are $k$-independent. So $\sum_{i} a_{i} \# w_{i}=0$, which is the same as $\sum_{i} a_{i} \otimes_{k} w_{i}=0$, implies each $a_{i}=0$, that is, $f=\sum_{i} a_{i} w_{i}=0$. So the $k$-algebra homomorphism $R[X ; \phi] \rightarrow R \# k\langle X\rangle$ defined above is actually a $k$-algebra isomorphism, as expected.

Let $R$ be a ring with the center $C$, which forms a field. Clearly, $\delta(C) \subseteq C$ for any $\delta \in \operatorname{Der}(R)$. Given a map $\phi: X \rightarrow \operatorname{Der}(R)$, where $X$ is a set of indeterminates, write $\delta_{x} \stackrel{\text { def. }}{=} \phi(x)$ for $x \in X$ and define

$$
C^{(\phi)} \stackrel{\text { def. }}{=}\left\{\alpha \in C: \delta_{x}(\alpha)=0 \text { for any } x \in X\right\} \text {. }
$$

Clearly, $C^{(\phi)}$ is a subfield of $C$. Let $k$ be any subfield of $C^{(\phi)}$. The simplest choice of $k$ is the prime subfield of $C$. Then $\phi(x) \stackrel{\text { def. }}{=} \delta_{x} \in \operatorname{Der}_{k}(R)$ for $x \in X$. By Lemma 4. $R[X ; \phi]$ is canonically isomorphic to the smash product $R \# k\langle X\rangle$. With this, we are able to apply Theorems 2 and 3 to the Ore extension $R[X ; \phi]$. For the convenience of later applications, we recall Definition 4 and Theorems 2 and 3 in the context of Ore extensions as follows.

Definition 5. Let $R$ be a ring with the center $C$ being a field. In the Ore extension $R[X ; \phi]$, let $\mathfrak{g}$ be the free Lie algebra generated by $X$ over the prime field of $C$ and let $h \in C \mathfrak{g} \mapsto \delta_{h} \in \operatorname{Der}(R)$ be the left $C$-linear Lie map extending the map $\phi: X \rightarrow \operatorname{Der}(R)$. Let $\mathfrak{g}_{0}$ be the $C$-space of $h \in \mathfrak{g}$ such that $\delta_{h} \in \operatorname{Der}_{0}(R)$. Let $B$ be a $C$-basis of $C \mathfrak{g}$ such that $B_{0} \stackrel{\text { def. }}{=} B \cap \mathfrak{g}_{0}$ forms a $C$-basis of $\mathfrak{g}_{0}$. For $h \in B_{0}$, choose $a_{h} \in R$ such that $\delta_{h}=\operatorname{ad}\left(a_{h}\right)$. Define $B_{0}^{\prime} \stackrel{\text { def. }}{=}\left\{h-a_{h}: h \in B_{0}\right\}$ and $B^{\prime} \stackrel{\text { def. }}{=} B_{0}^{\prime} \cup\left(B-B_{0}\right)$. Let $<$ be a linear order of $B^{\prime}$ such that $h<g$ for $h \in B_{0}^{\prime}$ and $g \in B-B_{0}$. We call $B^{\prime}$ so ordered a regular Lie basis of the Ore extension $R[X ; \phi]$.

Theorem 5. Let $R$ be a ring with the center $C$ being a field. Set $S \stackrel{\text { def. }}{=} R[X ; \phi]$. Let $B^{\prime}$ be a regular Lie basis of $S$ and retain the notation of Definition 5 . We have the following:

(1) Regular $B^{\prime}$-words form an $R$-basis of $R[X ; \phi]$.

(2) For $T \subseteq R, C_{S}(T)=C_{S}(R)$ iff for any $\delta \in C \phi(\mathfrak{g})+\operatorname{Der}_{0}(R), \delta(T)=0$ implies $\delta(R)=0$. 
(3) The set of regular $B_{0}^{\prime}$-words forms a $C$-basis of the free $C$-module $C_{S}(R)$. For any $C$-basis $V$ of $R$, the set

$$
\mathbb{B} \stackrel{\text { def. }}{=}\left\{v W: v \in V \text { and } W \text { is a regular word in } B-B_{0}\right\}
$$

forms a $C_{S}(R)$-basis of the free $C_{S}(R)$-module $S$.

(4) Any element of $S \otimes_{C_{S}(R)} S$ can be uniquely expressed in the form $\sum_{i} f_{i} \otimes g_{i}$, where $f_{i} \in S$ and where $g_{i} \in \mathbb{B}$ are distinct.

Proof. (1) follows by Theorem 2 and the rest by Theorem 3

It is interesting to see the special instance of Theorem 5 for $X=\{x\}$. This has already generalized all the known results in the literature, in which $R$ has to be simple [1] or prime [9]. Ours is true for any ring $R$ with the center $C$ being a field.

Corollary 6. Consider the Ore extension $S \stackrel{\text { def. }}{=} R[x ; \delta]$, where $\delta \in \operatorname{Der}(R)$ and where $R$ is a ring with the center $C$ being a field. Set $C^{(\delta)} \stackrel{\text { def. }}{=}\{\alpha \in C: \delta(\alpha)=0\}$. Let $Z_{S}$ denote the center of $S$.

(1) $\operatorname{char} R=0$ : If $\delta$ is inner, say $\delta=\operatorname{ad}(a)$, where $a \in R$, then $C_{S}(R)=Z_{S}=$ $C[\xi]$, where $\xi \stackrel{\text { def. }}{=} x-a$. Otherwise, $C_{S}(R)=C$ and $Z_{S}=C^{(\delta)}$.

(2) char $R=p \geq 2$ : Assume that there exist $\alpha_{i} \in C^{(\delta)}$ and $a \in R$ such that

$$
\delta^{p^{s}}+\alpha_{1} \delta^{p^{s-1}}+\cdots+\alpha_{s} \delta=\operatorname{ad}(a) .
$$

Let $s \geq 0$ above be the minimal such integer. Then $C_{S}(R)=C[\xi]$, where $\xi \stackrel{\text { def. }}{=}$ $x^{p^{s}}+\alpha_{1} x^{p^{s-1}}+\cdots+\alpha_{s} x-a$. If $\delta(a) \in \delta(C)$, say $\delta(a)=\delta(\alpha)$, where $\alpha \in C$, then $Z_{S}=C^{(\delta)}[\xi+\alpha]$. If $\delta(a) \notin \delta(C)$, then $Z_{S}=C^{(\delta)}\left[\xi^{p}\right]$. If $\delta$ does not satisfy any identities of the form $(\dagger)$, then $C_{S}(R)=C$ and $Z_{S}=C^{(\delta)}$.

Proof. We retain the notation of Theorem 5 Let $k$ be the prime field of $C$. For the case char $R=0$, the Lie ring $\mathfrak{g}$ generated by $k x$ is $k x$ itself. If $\delta=\operatorname{ad}(a)$ for some $a \in R$, then $\mathfrak{g}_{0}=\mathfrak{g}=C x$ and $C_{S}(R)=C[\xi]$, where $\xi \stackrel{\text { def. }}{=} x-a$, follows from Theorem 5 by letting $B_{0}^{\prime} \stackrel{\text { def. }}{=}\{\xi\}$. Since $C_{S}(R) \supseteq Z_{S} \supseteq C[\xi]$, we have $C[\xi]=Z_{S}$. If $\delta$ is outer, then $\mathfrak{g}_{0}=0$ and $C_{S}(R)=C$ follows from Theorem 5 by letting $B_{0}^{\prime} \stackrel{\text { def. }}{=} \emptyset$. For the case char $R=p \geq 2$, the Lie ring $\mathfrak{g}$ generated by $k x$ is $\bigoplus_{0 \leq j} k x^{p^{j}}$, and $C \mathfrak{g}=\bigoplus_{0 \leq j} C x^{p^{j}}$, the left $C$-space spanned by $\left\{x^{p^{j}}: j \geq 0\right\}$. Suppose that $\delta$ satisfies $(\dagger)$ with $s$ being the minimum. Set $y \stackrel{\text { def. }}{=} x^{p^{s}}+\alpha_{1} x^{p^{s-1}}+\cdots+\alpha_{s} x$. Clearly,

$$
\mathfrak{g}=\bigoplus_{0 \leq j} C x^{p^{j}}=\bigoplus_{0 \leq j<s} C x^{p^{j}} \oplus \bigoplus_{0 \leq t} C y^{p^{t}}
$$

By the minimality of $s, \mathfrak{g}_{0}=\bigoplus_{0 \leq t} C y^{p^{t}}$. So $\mathfrak{g}_{0}$ has the left $C$-basis $B_{0} \stackrel{\text { def. }}{=}\left\{y^{p^{t}}\right.$ : $t \geq 0\}$. Clearly, $\delta_{y^{p^{t}}}=\operatorname{ad}\left(a^{p^{t}}\right)$. Set $B_{0}^{\prime} \stackrel{\text { def. }}{=}\left\{y^{p^{t}}-a^{p^{t}}: t \geq 0\right\}$ and $\xi \stackrel{\text { def. }}{=} y-a$. Since $y a=a y$, we have $y^{p^{t}}-a^{p^{t}}=\xi^{p^{t}}$. So $B_{0}^{\prime}=\left\{\xi^{p^{t}}: t \geq 0\right\}$. Order $B_{0}^{\prime}$ linearly by setting $\xi<\xi^{p}<\xi^{p^{2}}<\cdots$. Regular $B_{0}^{\prime}$ words consist of $\xi^{n}, n \geq 0$. So $C_{S}(R)=C[\xi]$ follows by Theorem [5. Since $S$ is generated by $R$ and $x$, we have $Z_{S}=\left\{f \in C_{S}(R):[f, x]=0\right\}$. For $\alpha \in C, \delta \alpha=\alpha \delta+\delta(\alpha)$. With this, we multiply 
$(\dagger)$ by $\delta$ from the left-hand sides, from the right-hand sides, and then take their difference. This yields

$$
\delta\left(\alpha_{1}\right) \delta^{p^{s-1}}+\cdots+\delta\left(\alpha_{s}\right) \delta=\operatorname{ad}(\delta(a)) .
$$

This implies $\delta\left(\alpha_{i}\right)=0$ and $\delta(a) \in C$ by the minimality of $s$. With this,

$$
[\xi, x]=[y-a, x]=\delta\left(\alpha_{1}\right) x^{p^{s-1}}+\cdots+\delta\left(\alpha_{s}\right) x-\delta(a)=-\delta(a) .
$$

Assume $\delta(a) \in \delta(C)$, say $\delta(a)=\delta(\alpha)$, where $\alpha \in C$. Then $[\xi+\alpha, x]=-\delta(a)+$ $\delta(\alpha)=0$, implying $\xi+\alpha \in Z_{S}$. Since $C_{S}(R)=C[\xi]=C[\xi+\alpha], Z_{S}=C^{(\delta)}[\xi+\alpha]$. Assume $\delta(a) \notin \delta(C)$. Since $\delta(a) \in C$, we have $\left[\xi^{p}, x\right]=p \xi^{p-1} \delta(a)=0$. Any $f \in C_{S}(R)$ can be written uniquely in the form

$$
f=a_{0}(\xi)+a_{1}(\xi) \xi^{p}+a_{2}(\xi) \xi^{p^{2}}+\cdots,
$$

where each $a_{i}(\xi) \in C[\xi]$ has $\xi$-degree $<p$. So $f \in Z_{S}$ iff $0=[f, x]=\left[a_{0}(\xi), x\right]+$ $\left[a_{1}(\xi), x\right] \xi^{p}+\left[a_{2}(\xi), x\right] \xi^{p^{2}}+\cdots$, iff $\left[a_{i}(\xi), x\right]=0$ for each $i$. Write $a_{i}(\xi)=\sum_{j=0}^{t} \beta_{j} \xi^{j}$, where $0 \leq j \leq t<p, \beta_{j} \in C$ and $\beta_{t} \neq 0$. We have

$$
\begin{aligned}
{\left[a_{i}(\xi), x\right] } & =\sum_{j=0}^{t}\left[\beta_{j}, x\right] \xi^{j}+\sum_{j=1}^{t} \beta_{j}\left[\xi^{j}, x\right] \\
& =-\sum_{j=0}^{t} \delta\left(\beta_{j}\right) \xi^{j}-\sum_{j=1}^{t} j \delta(a) \beta_{j} \xi^{j-1} \\
& =-\sum_{j=0}^{t-1}\left(\delta\left(\beta_{j}\right)+(j+1) \delta(a) \beta_{j+1}\right) \xi^{j}-\left(\delta\left(\beta_{t}\right)\right) \xi^{t} .
\end{aligned}
$$

Suppose $\left[a_{i}(\xi), x\right]=0$. Then $\delta\left(\beta_{t}\right)=0$ and $\delta\left(\beta_{t-1}\right)+t \delta(a) \beta_{t}=0$. If $t>0$, then $\delta(a)=\frac{-\delta\left(\beta_{t-1}\right)}{t \beta_{t}}=-\delta\left(\frac{\beta_{t-1}}{t \beta_{t}}\right) \in \delta(C)$, contradicting our assumption. So $t=0$ and $a_{i}(\xi)=\beta_{0} \in C^{(\delta)}$. We have thus shown that $f \in Z_{S}$ implies $f \in C^{(\delta)}\left[\xi^{p}\right]$. Clearly, $f \in C^{(\delta)}\left[\xi^{p}\right]$ implies $f \in Z_{S}$. So $Z_{S}=C^{(\delta)}\left[\xi^{p}\right]$ follows.

\section{Differential identities}

A differential identity of $R$ is an equality

$$
\sum_{i} \sum_{j} a_{i j} w_{i}(r) b_{i j}=0 \quad \forall r \in R,
$$

where $a_{i j}, b_{i j} \in R$ and where $w_{i}$ are compositions of derivations of $R$. In the Ore extension $R[X ; \phi]$, write $\phi(x)=\delta_{x}$ for $x \in X$. For $r \in R$,

$$
\begin{aligned}
\delta_{x}(r) & =[x, r] \stackrel{\text { def. }}{=} x r-r x, \\
\delta_{y} \delta_{x}(r) & =[y,[x, r]]
\end{aligned}
$$

In this way, a differential identity involving derivations in $\phi(X)$ can be put in the form

$$
\sum_{i} f_{i} r g_{i}=0 \quad \forall r \in R,
$$

where $f_{i}, g_{i} \in R[X ; \phi]$. We will prove the following. 
Theorem 7. Let $R$ be a prime ring and $Q$ its symmetric Martindale quotient ring. Set $S \stackrel{\text { def. }}{=} Q[X ; \phi]$ and

$$
C_{S}(R) \stackrel{\text { def. }}{=}\{f \in S: f r=r f \text { for } r \in R\} .
$$

Given $f_{i}, g_{i} \in S, \sum_{i} f_{i} r g_{i}=0$ for all $r \in R$ iff $\sum_{i} f_{i} \underset{C_{S}(R)}{\otimes} g_{i}=0$.

We recall some notation from [6] and [7]. Let $Q^{\text {op }}$ denote the opposite ring of $Q$ and let $\mathbb{Z}$ be the ring of integers. The tensor product $Q \otimes_{\mathbb{Z}} Q^{\text {op }}$ consists of elements in the form $\sum_{i} r_{i} \otimes r_{i}^{\prime}$, where $r_{i} \in Q$ and $r_{i}^{\prime} \in Q^{\text {op }}$. For $f \in S$ and $\beta=\sum_{i} r_{i} \otimes r_{i}^{\prime} \in Q \otimes_{\mathbb{Z}} Q^{\text {op }}$, we define

$$
f \cdot \beta \stackrel{\text { def. }}{=} \sum_{i} r_{i}^{\prime} f r_{i}
$$

Let $L$ denote the subring of $Q \otimes_{\mathbb{Z}} Q^{\text {op }}$ generated by the elements of the form $r \otimes r^{\prime}$ for all $r, r^{\prime} \in R \cup\{1\}$. Thus we can regard $S$ as a right $L$-module. For a subset $Y \subseteq S$, we define

$$
Y^{\perp} \stackrel{\text { def. }}{=}\{\beta \in L \mid f \cdot \beta=0 \text { for all } f \in Y\} .
$$

Note that $Y^{\perp}$ is an $(R, R)$-submodule of $L$. On the other hand, for $U \subseteq L$, we define

$$
U^{\perp} \stackrel{\text { def. }}{=}\{f \in S \mid f \cdot \beta=0 \text { for all } \beta \in U\} .
$$

We need the following.

Lemma 8 (Lemma 4 [5]). Let $C$ denote the extended centroid of $R$. Given finitely many $a_{1}, \ldots, a_{n} \in Q$, we have

$$
\left(C a_{1}+\cdots+C a_{n}\right)^{\perp \perp}=a_{1} C_{S}(R)+\cdots+a_{n} C_{S}(R) .
$$

We are ready for

Proof of Theorem 17. The implication $\Leftarrow$ is obvious. For the implication $\Rightarrow$, we apply Theorem 5. It is well known that any derivation of $R$ can be uniquely extended to $Q$. So any derivation of $Q$ vanishing on $R$ must also vanish on $Q$. So $C_{S}(R)=C_{S}(Q)$ by Theorem 5 , Let $C$ denote the extended centroid of $R$, which is defined to be the center of $Q$. Fix a $C$-basis $V$ of $Q$. By Theorem 5 , the set of regular $B^{\prime}$-words forms a right $Q$-basis of $S$, the set of regular $B_{0}^{\prime}$-words forms a $C$-basis of $C_{S}(R)$ and the set

$$
\mathbb{B} \stackrel{\text { def. }}{=}\left\{v U: v \in V \text { and } U \text { is a regular word in } B-B_{0}\right\}
$$

forms a basis of the free $C_{S}(R)$-module $S$. Let $g_{1}, g_{2}, \ldots$ enumerate elements of $\mathbb{B}$. By (4) of Theorem [5, we have for any $f_{i} \in S$,

$$
\sum_{i} f_{i} \underset{C_{S}(R)}{\otimes} g_{i}=0 \Leftrightarrow \text { all } f_{i}=0 .
$$

Assume on the contrary that there exist $0 \neq f_{i} \in Q[X ; \phi]$, and $g_{i} \in \mathbb{B}$ such that

$$
\sum_{i} f_{i} r g_{i}=0 \quad \text { for all } r \in R .
$$

Fix arbitrarily a linear order $\preceq$ of $X$-words such that

$$
\text { short word } \prec \text { long word. }
$$


The $\preceq$-leading word of $0 \neq f \in S$ is the $\preceq$-maximal word occurring nontrivially in $f$. By the $\preceq$-leading word of $(\ddagger)$, we mean the $\preceq$-maximum of $\preceq$-leading words of nonzero $f_{i}$ 's. We may further choose $(\ddagger)$ so that that its $\preceq$-leading word $W$ is minimal possible. For each $i$, write

$$
f_{i}=a_{i} W+\cdots .
$$

For $\beta \in \bigcap_{i} a_{i}^{\perp}$, each $f_{i} \cdot \beta$ has $\preceq$-leading word $<W$. We easily see $\sum_{i}\left(f_{i} \cdot \beta\right) r g_{i}=0$ for all $r \in R$. By the $\preceq$-minimality of $(\ddagger)$, we have $f_{i} \cdot \beta=0$. So $\beta \in \bigcap_{i} a_{i}^{\perp}$ implies $f_{i} \cdot \beta=0$. By Lemma $8, f_{i} \in \sum_{j} a_{j} C_{S}(R)$. Let $\xi_{j}$ enumerate regular $B_{0}^{\prime}$-words. We may thus write

$$
f_{i}=\sum_{j} b_{i j} \xi_{j}, \quad \text { where } b_{i j} \in Q
$$

With this, rewrite $(\ddagger)$ as

$$
0=\sum_{i} \sum_{j} b_{i j} \xi_{j} r g_{i}=\sum_{i} \sum_{j} b_{i j} r \xi_{j} g_{i} \quad \text { for all } r \in R .
$$

Let $v_{1}, v_{2}, \ldots$ enumerate $V$ and $U_{1}, U_{2}, \ldots$ enumerate regular $B^{\prime}$-words. Each $g_{i} \in \mathbb{B}$ can be written uniquely as $g_{i}=v_{s} U_{t}$. We re-index the corresponding $b_{i j}$ as $b_{s t j}$ if $g_{i}=v_{s} U_{t}$. So

$$
0=\sum_{s, t} \sum_{j} b_{s t j} r \xi_{j} v_{s} U_{t}=\sum_{s, t, j} b_{s t j} r v_{s} \xi_{j} U_{t} \quad \text { for all } r \in R .
$$

Distinct ordered pairs $(j, t)$ correspond to distinct regular $B^{\prime}$-words $\xi_{j} U_{t}$ and these regular $B^{\prime}$-words form a right $R$-basis of $S$. So for a fixed ordered pair $(j, t)$,

$$
0=\sum_{s} b_{s t j} r v_{s}
$$

But $V=\left\{v_{1}, v_{2}, \ldots\right\}$ forms a $C$-basis of $Q$. By the well-known Martindale's lemma, $b_{s t j}=0$. This is true for all $s, t, j$. So all $b_{i j}=0$ and hence $f_{i}=\sum_{j} b_{i j} \xi_{j}=0$ for all $i$, contradicting the assumption.

It is interesting to deduce Kharchenko's Theorem from the above.

Theorem 9 (Lemma 2 [6]). Let $R$ be a prime ring, $Q$ its symmetric Martindale quotient ring and $C$ its extended centroid. Suppose that $\delta_{i} \in \operatorname{Der}(Q), i=1,2, \ldots$, are mutually outer in the sense that given any finite sum $\sum_{i} \alpha_{i} \delta_{i} \in \operatorname{Der}_{0}(Q)$, where $\alpha_{i} \in C$, then all $\alpha_{i}=0$ follows. Let $<$ linearly order these $\delta_{i}$. Let $w_{j}$ enumerate regular words in these $\delta_{i}$. Given $a_{i j}, b_{i j} \in Q$, if

$$
\sum_{i, j \geq 0} a_{i j} w_{j}(r) b_{i j}=0 \quad \text { for all } r \in R,
$$

then $\sum_{i} a_{i j} \underset{C}{\otimes} b_{i j}=0$ for each $j$.

Proof. Pick a set $X=\left\{x_{1}, x_{2}, \ldots\right\}$ of indeterminates with the cardinality equal to that of these $\delta_{i}$. Define $\phi: X \rightarrow \operatorname{Der}(Q)$ such that $\phi\left(x_{i}\right) \stackrel{\text { def. }}{=} \delta_{i}$. Extend $\phi$ to $X$-words by setting

$$
\phi\left(x_{i_{1}} x_{i_{2}} \cdots\right) \stackrel{\text { def. }}{=} \phi\left(x_{i_{1}}\right) \phi\left(x_{i_{2}}\right) \cdots
$$


where $x_{i_{1}}, x_{i_{2}}, \ldots \in X$. Conversely, given a product (or word) $w$ of derivations in $\operatorname{Der}(Q)$, let $\phi^{-1}(w)$ be the $X$-word $W$ such that $\phi(W)=w$. Let $k$ be the prime subfield of $C$ and $\mathfrak{g}$ the free Lie algebra generated by $X$ over $k$. Define

$$
\mathfrak{g}_{0}=\left\{h \in C \mathfrak{g}: \phi(h) \in \operatorname{Der}_{0}(Q)\right\}
$$

Fix a $C$-basis $B_{0}$ of $\mathfrak{g}_{0}$. The set $X \cup B_{0}$ is $C$-independent by the mutual outerness of $\delta_{i} \stackrel{\text { def. }}{=} \phi\left(x_{i}\right)$ and hence can be extended to a left $C$-basis $B$ of $C \mathfrak{g}$. For $h \in B_{0}$, pick $a_{h} \in Q$ such that $\phi(h)=\operatorname{ad}\left(a_{h}\right)$. Set $B_{0}^{\prime} \stackrel{\text { def. }}{=}\left\{h-a_{h}\right\}$. Order $X$ by setting $x_{i}<x_{j}$ if $\delta_{i}<\delta_{j}$. Extend this order of $X$ to $B^{\prime}$ such that $h<g$ for $h \in B_{0}^{\prime}$ and $g \in B-B_{0}$. Given a derivation word $w=\cdots \delta_{s_{2}} \delta_{s_{1}}$, where $\delta_{s_{i}} \in \operatorname{Der}(Q)$, write

$$
w(r)=\left[\cdots \cdots\left[x_{s_{2}},\left[x_{s_{1}}, r\right]\right] \cdots\right]=\sum_{l} f_{l} r g_{l}
$$

where $f_{l}, g_{l}$ are subwords of the word $\phi^{-1}(w)=\cdots x_{s_{2}} x_{s_{1}}$. So if $w$ is a regular word in derivations, then $f_{l}, g_{l}$ are regular $X$-words (and hence regular $\left(B-B_{0}\right)$-words, since $\left.X \subseteq B-B_{0}\right)$. Given $a_{i j}, b_{i j} \in Q$, suppose that

$$
\sum_{i, j} a_{i j} w_{j}(r) b_{i j}=0 \quad \text { for all } r \in R
$$

In the way explained above, write this in the form $\sum_{l} f_{l} r g_{l}=0$, where $f_{l}, g_{l} \in S$ involve only regular $X$-subwords of $\phi^{-1}\left(w_{j}\right)$. By Theorem $7 \sum_{l} f_{l} \otimes g_{l}=0$, where $\otimes$ is taken over $C_{S}(R)$. Since $f_{l}, g_{l} \in S$ involve only regular $X$-subwords of $\phi^{-1}\left(w_{j}\right)$, $\sum_{l} f_{l} \otimes g_{l}$ is a sum of terms of the form

$$
a W \otimes b W^{\prime},
$$

where $a, b \in Q$ and where $W, W^{\prime}$ are regular $X$-subwords of some $\phi^{-1}\left(w_{j}\right)$. We may assume that $w_{0}$ has the maximal length $n$ among all $w_{i}$. In $\sum_{l} f_{l} \otimes g_{l}$, the sum of terms involving $W_{0} \stackrel{\text { def. }}{=} \phi^{-1}\left(w_{0}\right)$ is clearly

$$
\sum_{i} a_{i 0} W_{0} \otimes b_{i 0}+(-1)^{n} \sum_{i} a_{i 0} \otimes b_{i 0} W_{0} .
$$

By (4) of Theorem 5, $\sum_{i} a_{i 0} \otimes_{C} b_{i 0}=0$. On the other hand, $\sum_{i} a_{i 0} \otimes_{C} b_{i 0}=0$ implies $\sum_{i} a_{i 0} w_{0}(r) b_{i 0}=0$ for $r \in R$. So we have

$$
\sum_{i, j>0} a_{i j} w_{j}(r) b_{i j}=0 \quad \text { for all } r \in R .
$$

Apply the same argument to this and continue in this manner. It follows that $\sum_{i} a_{i j} \otimes_{C} b_{i j}=0$ for all $j$.

\section{REFERENCES}

[1] S. A. Amitsur, Derivations in simple rings, Proc. London Math. Soc. (3) 7 (1957), 87-112. MR0088480 (19:525d)

[2] K. I. Beidar, W. S. Martindale, III, A. V. Mikhalev, "Rings with generalized identities", Monographs and Textbooks in Pure and Applied Mathematics 196, Marcel Dekker, Inc., New York, 1996. MR1368853 (97g:16035)

[3] V. D. Burkov, On differentially prime rings, (Russian) Uspekhi Mat. Nauk 35(5) (1980), 219-220. (Engl. Transl. Russian Math. Surveys 35(5):253-254.) MR595145 (82f:16002)

[4] C.-L. Chuang and Y.-T. Tsai, Higher derivations of Ore extensions by q-skew derivations, Journal of Pure and Applied Algebra, 214(10) (2010), 1778-1786. MR 2608105

[5] C.-L. Chuang, T.-K. Lee, C.-K. Liu and Y.-T. Tsai, Higher Derivations of Ore Extensions, Israel J. Math 175 (2010), 157-178. MR2607542 
[6] V. K. Kharchenko, Differential identities of prime rings, (Russian) Algebra i Logika 17(2) (1978), 220-238. (Engl. Transl., Algebra and Logic 17(2) (1978), 154-168.) MR541758 (81f:16025)

[7] V. K. Kharchenko, Differential identities of semiprime rings, (Russian) Algebra i Logika 18(1) (1979), 86-119. (Engl. Transl., Algebra and Logic 18(1) (1979), 58-80.) MR566776 (81f:16052)

[8] A. Leroy and J. Matczuk, The extended centroid and X-inner automorphisms of Ore extensions, J. Algebra 145(1) (1992), 143-177. MR1144664 (93b:16053)

[9] J. Matczuk, Extended centroids of skew polynomial rings, Math. J. Okayama Univ. 30 (1988), 13-20. MR976726 (89m:16006)

[10] S. Montgomery, "Hopf algebras and their actions on rings", Regional conference series in mathematics; no. 82, American Mathematical Society, Providence, Rhode Island, 1992. MR.1243637 (94i:16019)

[11] C. Reutenauer, "Free Lie algebras", London Mathematical Society monographs; new ser. 7, Oxford: Clarendon Press; New York, Oxford University Press, 1993. MR.1231799 (94j:17002)

[12] J.-P. Serre, "Lie algebras and Lie groups: 1964 lectures given at Harvard University", (1992), 2nd ed., Lecture Notes in Mathematics, 1500, Springer-Verlag: Berlin. MR 1176100 (93h:17001)

[13] M. E. Sweedler, "Hopf Algebras", Mathematics Lecture Notes Series, 1969, W. A. Benjamin, Inc., New York, 1996. MR0252485 (40:5705)

[14] Y.-T. Tsai and C.-L. Chuang, Quotient Rings of Ore Extensions with More Than One Indeterminate, Commun. Algebra 36(10), (2008), 3608-3615. MR2458396 (2009k:16054)

[15] Y.-T. Tsai, T.-Y. Wu, and C.-L. Chuang, Jacobson radicals of Ore extensions of derivation type, Commun. Algebra 35(3) (2007), 975-982. MR2305244(2007m:16045)

Department of Mathematics, National Taiwan University, Taipei 106, Taiwan

E-mail address: chuang@math.ntu.edu.tw

Department of Applied Mathematics, Tatung University, Taipei 104, Taiwan

E-mail address: yttsai@ttu.edu.tw 